\title{
A Survey on Various Techniques of Super Resolution Imaging
}

\author{
Robinson Macwan \\ Department of Information \\ Technology, \\ CHARUSAT University, \\ India
}

\author{
Nehal Patel \\ Department of Information \\ Technology, \\ CHARUSAT University, \\ India
}

\author{
Priteshkumar Prajapati \\ Department of Information \\ Technology, \\ CHARUSAT University, \\ India
}

\author{
Jaimin Chavda \\ Department of Information \\ Technology, \\ CHARUSAT University, \\ India
}

\begin{abstract}
Resolution plays a major role for interpretation and analysis of an image. Super Resolution is a technique to enhance the resolution of an image from single or multiple low resolved images, which gives detailed information present in an image. In this paper, we describe several methods for Super Resolution (SR) that enhances the quality of an image. Mainly the methods are divided into frequency domain and spatial domains. Here, we stated comparison of different approaches, challenges and issues for SR and applications of SR in practical world e.g. in medical imaging, satellite imaging, and forensics.
\end{abstract}

\section{General Terms}

Low Resolution, High Resolution, Super Resolution

\section{Keywords}

Low Resolution, High Resolution, Super Resolution, Interpolation.

\section{INTRODUCTION}

In most image processing applications, an image is required to be highly resolved. It is mainly required for better pictorial view for human interpretation or for machine perception for making better decisions. Resolution is mainly concerned with the number of pixels per inch (ppi) or dots per inch (dpi) the image possesses. If the number of pixels is less than the image produces will be of low resolution (LR) and offers very less information. As the pixel density increases the image quality as well as the information offering by the image increases. But as the number of pixels increases the light on the sensors decreases which results in shot noise.

Usually, the sensor limits the quality of an image due to its physical characteristics like size and density of detectors [2].

The degradations in an image quality are caused at the recording process such as optical distortion, motion blur caused by limited shutter speed, noise and aliasing effects.

Figure 1 shows the common image acquisition system, where different factor affects the image quality like over the air (OTA), charge-couple device (CCD), preprocessors and environment. Optical Blur is a non-symmetric design of the lens and an aperture before or behind the optic center of the lens lead to image distortions. Motions blur results when the image being recorded changes during the recording of a single frame, either due to rapid movement or long exposure. Noise in an image is an undesirable by-product of image capture that adds spurious and extraneous information.

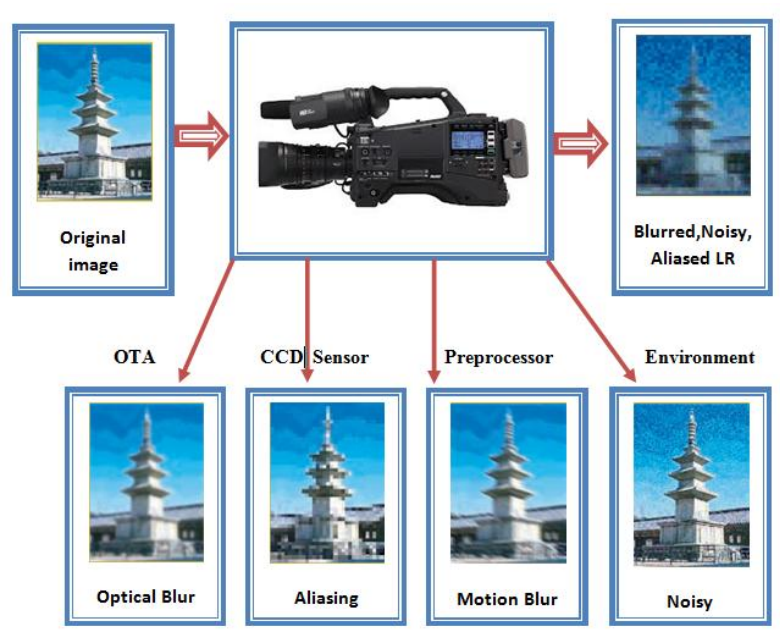

Figure 1: Common image acquisition system

Aliasing effects refer to an effect which can create confusion between different signals when sampled. Due to these, the final observed image is blurred and noisy.

One way of producing a high resolution (HR) image, is by installing a high resolution sensor. But it is not very feasible to do so. It results in increase of a cost as well as increase in power consumption. A simple example of this is satellite imaging system or a medical imaging system, where it is infeasible to use a high resolution sensor. So, to come over these, post processing is required to develop a better resolved image that holds more information. One of the promising approaches for this is signal processing techniques to obtain HR image from multiple LR images. Nowadays such approach is more active in research area, and is called Super Resolution (SR) or Resolution Enhancement [2].

\section{IMAGE SUPER RESOLUTION}

Super Resolution is a technique that enhances the image resolution and makes it clearer for human as well as for machines in view for better information extraction which are not visibly cleared in LR image. Super resolution can be acquired either by processing multiple low resolved images as input and generating a high detail containing a single super resolved image as output or enhancing the details in a single low resolved image and generating a high resolved image for analysis. In SR from multiple LR images, it is a construction of HR image from several LR images, thereby increasing the high frequency components. The basic idea behind this is to combine non-repetitive information contained by multiple LR images. While in SR from single LR image, resolution of the image can be increased either by enhancing the edges of the 
objects present in an image or by patch redundancy technique, where each LR patch is replaced by its corresponding HR patch.

The main benefit of SR approach is that, a HR image can be obtained even with the existing LR imaging with lower cost and less power consumption.

In SR reconstruction from multiple LR images, the basic assumption is that the LR should have enough shifted in viewing the same scene. If LR has minor shift then the HR reconstructed image will not contain any new information. Suppose that four images are taken and one image out of four can be taken as reference and other be shifted horizontally, vertically or diagonally to a scale of half pixels. By taking that one image as reference, other three image pixels can be interleaved and a higher resolved image can be generated.

\begin{tabular}{|c|c|c|}
\hline$X$ & $X$ & $X$ \\
\hline$X$ & $X$ & $X$ \\
\hline$X$ & $X$ & $X$ \\
\hline
\end{tabular}

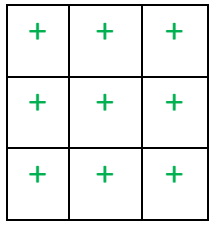

\begin{tabular}{|l|l|l|}
\hline 0 & 0 & 0 \\
\hline 0 & 0 & 0 \\
\hline 0 & 0 & 0 \\
\hline
\end{tabular}

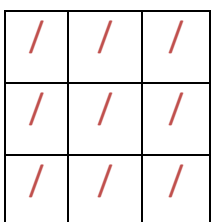

\begin{tabular}{|c|c|c|c|c|c|}
\hline $\mathrm{X}$ & + & $\mathrm{X}$ & + & $\mathrm{X}$ & + \\
\hline $\mathrm{O}$ & $/$ & $\mathrm{O}$ & $/$ & $\mathrm{O}$ & $\mathrm{I}$ \\
\hline $\mathrm{X}$ & + & $\mathrm{X}$ & + & $\mathrm{X}$ & + \\
\hline $\mathrm{O}$ & $/$ & $\mathrm{O}$ & $/$ & $\mathrm{O}$ & $\mathrm{I}$ \\
\hline $\mathrm{X}$ & + & $\mathrm{X}$ & + & $\mathrm{x}$ & + \\
\hline $\mathrm{O}$ & $/$ & $\mathrm{O}$ & $/$ & $\mathrm{O}$ & $/$ \\
\hline
\end{tabular}

Figure 2: Ideal Super Resolution setup.

(Out of the Four images are taken, first one can be taken as reference and other three images can be considered to be relative shifted to half a pixel in horizontal, vertical, and diagonal directions (left side-four images). These three image pixels can then be added to produce a high resolution image with increase in size of the image (right side-single image)) [1].

Usually, a super resolution method consists of the following basic processing steps: (1) Registration, (2) Interpolation and (3) Deblurring or noise removal.

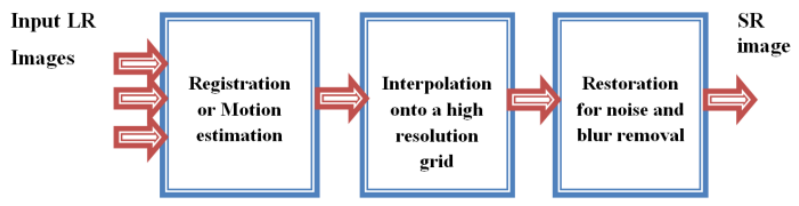

Figure 3: Basic SR reconstruction stages

Image registration is the process of overlapping more than one images of the same scene which has been taken from different angles by the sensors. In registration two or more images are align geometrically to obtain the information through image fusion or change detection.

Interpolation is a process of estimating the intermediate pixels between the pixel values. When any image is converted from LR to HR, intermediate gaps are introduced and these values have to be estimated and filled with interpolation process.

As the process of interpolation introduces some artifacts the resultant image will be blurred or noisy. Through different filters and techniques noise will be removed and finally a super resolved image is generated.

\section{APPROACHES TO SR}

Super-resolution techniques can be classified as (1) Frequency domain approach and (2) Spatial domain approach.

\subsection{Frequency domain approach}

Tsai and Huang proposed frequency based approach in which they stated to transform the LR image into Discrete Fourier Transform (DFT) domain and combined them according to the relationship between the aliased DFT coefficients of the observed LR images and that of the unknown high-resolution image. The combined data are then transformed back to the spatial domain where the new image could have a higher resolution than that of the input images [3]. The principles of frequency domain approach areas follow: i) what is the shift property of the Fourier transform? ii) The aliasing association between the continuous Fourier transform (CFT) of an original HR image and the discrete Fourier transform (DFT) of observed LR images, iii) the supposition that an original HR image is band limited. Through these properties system equation is derived [4].

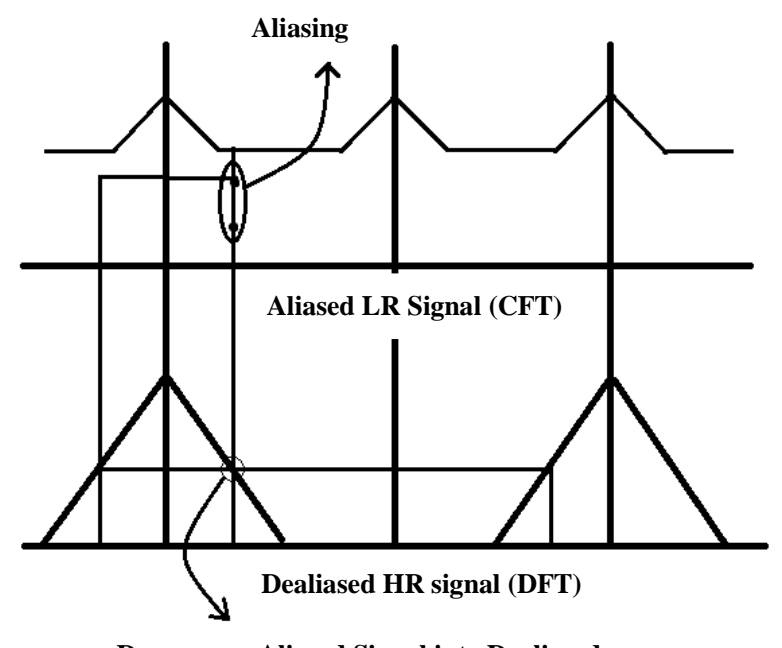

Decompose Aliased Signal into Dealiased

Figure 4: LR image relationship with HR image [4].

Frequency domain approach has some benefits like it is spontaneous way to enhance the details by extrapolating the high frequency information presented in LR images. As well it has lower computational complexity. But the disadvantage is that is incapable of handling the real-world applications.

\subsection{Spatial Domain Approach}

The frequency domain approach has certain drawbacks like it limits the inter-frame motion to be translational. As well it is very difficult in frequency domain to use the prior knowledge. As the main problem is ill-posed image in SR, prior knowledge is required to overcome this. The main benefit of spatial domain is the support for unbind motion between frames and prior knowledge availability for solving the problems. Some of the methods are interpolation, iterative back projection and projection onto convex [4]

\subsubsection{Interpolation}

Interpolation is the process of transferring image from one resolution to another without losing image quality. In Image processing field, image interpolation is very important function for doing zooming, enhancement of image, resizing any many more. Most common interpolation techniques are nearest neighbor, bilinear and cubic convolution. Digital image is a signal, spatially varying in two dimensions. This signal is sampled and quantized to get values. All these values 
called pixels of image. When we increase the resolution of image from low to high, it is called up-sampling or up-scaling while reverse is called down sampling or down scaling [5].

\section{(i). Bilinear interpolation}

In Bilinear interpolated point is filled with four closest pixel's weighted average.

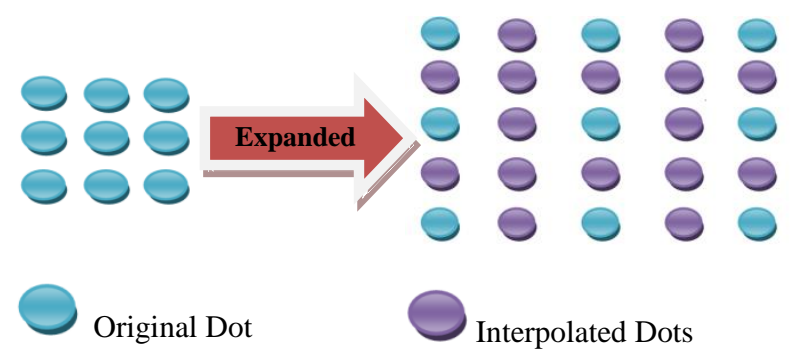

Figure 5: Basic concept of interpolation [5].

Bilinear interpolation is recommended for continuous data like elevation and raw slope values.

The interpolation kernel for linear interpolation is:

$$
u(x)= \begin{cases}0 & |x|>1 \\ 1-|x| & |x|<1\end{cases}
$$

Where $\mathrm{x}=$ distance between interpolated point and grid point [5].

\section{(ii). Bicubic interpolation}

Looks at the sixteen nearest cells and fits a smooth curve through the points to find the output value. Bicubic interpolation is recommended for smoothing continuous data, but this incurs a processing performance overhead.

The interpolation kernel for cubic interpolation is:

$$
u(x)=\left\{\begin{array}{cl}
\frac{3}{2}|x|^{3}-\frac{5}{2}|x|^{2}+1 & 0 \leq|x|<1 \\
-\frac{1}{2}|x|^{3}+\frac{5}{2}|x|^{2}-4|x|+2 & 1 \leq|x|<2 \\
0 & 2<|x|
\end{array}\right.
$$

Where $\mathrm{x}=$ distance between interpolated point and grid point [5].

\section{(iii). Nearest Neighbor interpolation}

In this method, nearest value is copied for interpolation and this technique has less computational complexity. Nearest neighbor interpolation is recommended for categorical data such as land use classification.

The interpolation kernel for each direction for this method is:

$$
u(x)= \begin{cases}0 & |x|>0.5 \\ 1 & |x|<0.5\end{cases}
$$

Where $\mathrm{x}=$ distance between interpolated point and grid point [5].

The resultant image produced by the interpolation technique generally suffers from artifacts.

\subsubsection{Iterative Back Projection (IBP)}

In IBP approach HR image is estimated by back projecting the difference between the simulated LR image and captured LR on interpolated image. This iterative process of SR does iterations until the minimization of the cost function is achieved. Mathematically the SR step to IBP is written as

$$
X=X^{(0)}+X_{e}
$$

Where, $X$ - interpolated image; $\mathrm{X}_{\mathrm{e}}$ - error correction [6].

\subsubsection{Classical Multi-Image SR}

In the classical multi-image SR, a set of LR images of the same scene is taken. If enough LR images became available then the equation is determined and a SR image is reconstructed. The assumption here is that the two or more LR images should contain distinguishable features. Because of these, practically it helps very less in improvement of image resolution, if distinguishable features in LR images are less [8].

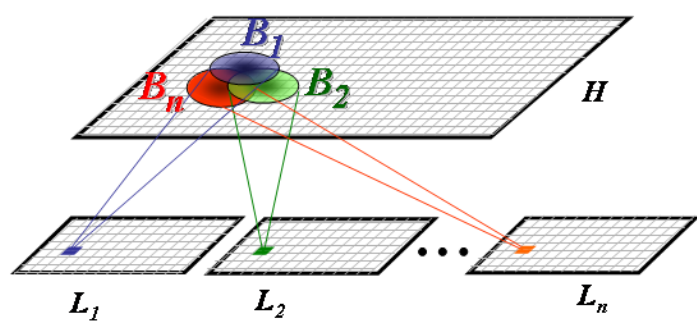

Figure 6: Multi-Image SR [8].

\subsubsection{Example-Based SR}

It is said that nature has repetitions in behave. In ExampleBased approach, the same rule is applied. This approach is useful when only single LR image is available. In this approach, the image has small patches that redundantly reappear, both within the scale as well as across the scale. Each LR patch in an image is replaced by its corresponding HR patch to generate the SR image. Here assumption is that, the image should have enough $\mathrm{HR}$ patches for the correspondence LR patches [8].

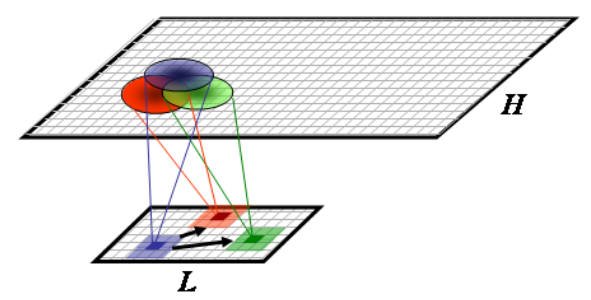

Figure 7: Single Image Multiple Patch [8].

\subsubsection{Learning Based SR}

It is a concept of machine learning, where the machine is trained to classify LR and its corresponding HR patches. In this approach, both LR and HR patches are divided into different classes. By doing so, the number of comparison reduces, as it has to compare LR with only HR patches.For an image if it is an edge-area of the LR, the routine examplebased image SR algorithm can be used to implement the local and fine SR. For the flat regions of the low-resolution, only interpolation algorithm is used for super-resolution.The performance of learning based super-resolution depends on HR patch(es) retrieved from the training data for an input LR patch [7]. 


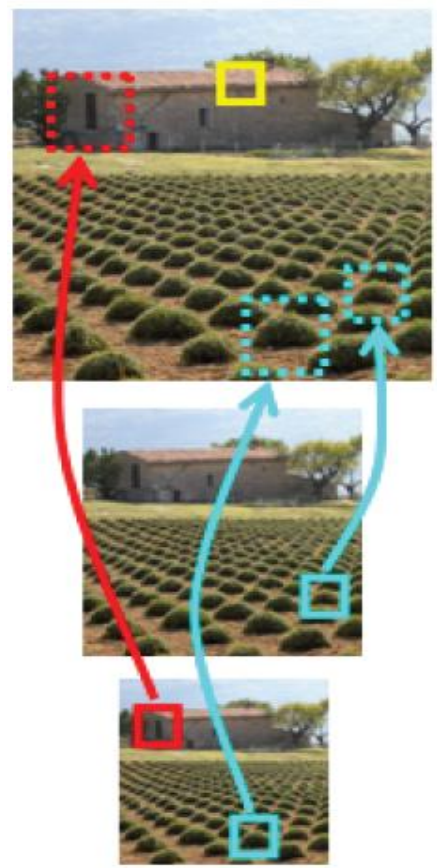

Figure 8: Patch recurrence within and across scales of a single image [8]

Dotted Squares are the HR patches for Solid Square LR patches as shown.

Table 1.Comparison of different SR approaches [9]

\begin{tabular}{|c|c|c|}
\hline Categorization & Description & Disadvantages \\
\hline $\begin{array}{c}\text { Interpolation } \\
\text { based }\end{array}$ & $\begin{array}{c}\text { Different } \\
\text { interpolation } \\
\text { techniques used }\end{array}$ & $\begin{array}{c}\text { Over-smooth, } \\
\text { jagged artifacts }\end{array}$ \\
\hline $\begin{array}{c}\text { Reconstruction } \\
\text { based }\end{array}$ & $\begin{array}{c}\text { Reconstruction } \\
\text { constraint and } \\
\text { image prior }\end{array}$ & $\begin{array}{c}\text { Ringing artifacts, } \\
\text { imposing additional } \\
\text { prior }\end{array}$ \\
\hline Learning based & $\begin{array}{c}\text { Learning high } \\
\text { frequency details } \\
\text { from training set }\end{array}$ & $\begin{array}{c}\text { High frequency } \\
\text { artifacts, relying } \\
\text { training set }\end{array}$ \\
\hline
\end{tabular}

\section{CHALLENGES ISSUES FOR SR}

In practical building SR image, there are several challenges and issues regarding that. Some of them are as follows:

\section{(i). Image Registration:}

In an image, image registration is a well known problem known by the name of ill-posed image. Image registration becomes more and more difficult when observed LR image is having very high aliasing effects. The registration error increases with decrease in the resolution of observed image. The degradation cased by these registration errors affects the quality of an image resolution more than that of interpolation [2].

(ii). Computational Efficiency:

Real time application is always requires good efficiency. As there are large numbers of unknowns in reconstructing SR images, matrix manipulation increases [10].

\section{(iii). Robustness:}

SR techniques are defenceless to the presence of outliers due to motion errors, inaccurate blur models, noise, moving objects, motion blur etc. These effects are not easy to estimate which are not acceptable in many applications like video conversions, so robustness of SR is required [10].

\section{APPLICATIONS}

Several practical areas and applications of SR as follows:

- Biometrics - Fingerprint recognition, Face recognition, Character recognition, DNA analysis.

- Medical Science - MRI, CT, X-Ray, Ultrasound [1].

- Satellite Imaging - Planetary information, Weather forecasting, Target detection, Traffic detection.

- Surveillance Video - Zooming region of interest (ROI). E.g. license plate recognition of vehicle, target recognition [1].

- Entertainment - HDTV, Photography.

- Commercial - Barcode reading.

- Military - Tracking and Detecting

\section{CONCLUSION}

This paper discusses about different techniques to achieve SR image from a single image or multiple LR images. We specified interpolation based, reconstruction based and learning based approaches to achieve the goal. We also include applications and comparison of different SR approaches. In future as per the application requirement such as in medical science and satellite imaging, using some of the above methods of SR, new methods can be derived by extending or integrating them which can generate more detailed containing SR images as result.

\section{REFERENCES}

[1] Sung Cheol Park, Min KyuPark, and Moon Gi Kang, 2003 Super-Resolution Image Reconstruction: A Technical Overview. IEEE Signal Processing Magazine.

[2] Pandya Hardeep, Prof. Prashant B. Swadas, Prof. Mahasweta Joshi, 2013,A Survey on Techniques and Challenges in Image Super Resolution Reconstruction. International Journal of Computer Science and Mobile Computing.

[3] Tsai and Huang, 2010. A survey on super-resolution imaging. Springer.

[4] R. SudheerBabu, Dr.K.E.Sreenivasa Murthy, 2011.A Survey on the Methods of Super-Resolution Image Reconstruction. International Journal of Computer Applications. Volume 15-No.2.

[5] Ankit Prajapati, Sapan Naik, Sheetal Mehta, 2012.Evaluation Of Different Image Interpolation Algorithms, International Journal of Computer Application. Volume 58-No.12.

[6] Rujul R Makwana, Nita D Mehta, 2013. Survey on Single Image Super Resolution Techniques. IOSR Journal of Electronics and Communication Engineering (IOSR-JECE). Volume 5, Issue 5.

[7] Amisha J. Shah1, Suryakant B. Gupta, 2012. Image Super Resolution - A Survey. IEEE.

[8] Daniel Glasner Shai Bagon Michal Irani, 2009. SuperResolution from a Single Image. IEEE.

[9] Qiang Zhou, Shifeng Chen, Jianzhuang Liu and Xiaoou Tang, 2011. Edge preserving single image Super Resolution. ACM.

[10] Jianchao Yang and Thomas Huang. Image superresolution: Historical overview and future challenges. University of illinois at Urbana-Champaign. 